\title{
A Xanthomonas oryzae pv. oryzae effector, XopR, associates with receptor-like cytoplasmic kinases and suppresses PAMP-triggered stomatal closure
}

\author{
Shuangfeng Wang ${ }^{1,2 \dagger}$, Jianhang Sun ${ }^{1,2 \dagger}$, Fenggui Fan ${ }^{1,2}$, Zhaoyun Tan ${ }^{1}$, Yanmin Zou ${ }^{1}$ \\ \& Dongping $\mathrm{Lu}^{1 *}$ \\ ${ }^{1}$ State Key Laboratory of Plant Genomics, Center for Agricultural Research Resources, Institute of Genetics and Developmental Biology, \\ Chinese Academy of Sciences, Shijiazhuang, Hebei 050021, China; \\ ${ }^{2}$ University of Chinese Academy of Sciences, Beijing 100049, China
}

Received May 16, 2016; accepted June 20, 2016; published online August 10, 2016

\begin{abstract}
Receptor-like kinases (RLKs) play important roles in plant immunity signaling; thus, many are hijacked by pathogen effectors to promote successful pathogenesis. Xanthomonas oryzae pv. oryzae (Xoo) is the causal agent of rice leaf blight disease. The strain PXO99A has 18 non-TAL (transcription activation-like) effectors; however, their mechanisms of action and host target proteins remain largely unknown. Although the effector XopR from the Xoo strain MAFF311018 was shown to suppress PAMP-triggered immune responses in Arabidopsis, its target has not yet been identified. Here, we show that PXO99A XopR interacts with BIK1 at the plasma membrane. BIK1 is a receptor-like cytoplasmic kinase (RLCK) belonging to the RLK family of proteins and mediates PAMP-triggered stomatal immunity. In turn, BIK1 phosphorylates XopR. Furthermore, XopR suppresses PAMP-triggered stomatal closure in transgenic Arabidopsis expressing XopR. In addition, XopR is able to associate with RLCKs other than BIK1. These results suggest that XopR likely suppresses plant immunity by targeting BIK1 and other RLCKs.
\end{abstract}

Xanthomonas, effector, XopR, BIK1, immunity

Citation: $\quad$ Wang, S., Sun, J., Fan, F., Tan, Z., Zou, Y., and Lu, D. (2016). A Xanthomonas oryzae pv. oryzae effector, XopR, associates with receptor-like cytoplasmic kinases and suppresses PAMP-triggered stomatal closure. Sci China Life Sci 59, 897-905. doi: 10.1007/s11427-016-5106-6

\section{INTRODUCTION}

As in animals, plants possess pattern-recognition receptors (PRRs) that recognize pathogen/microbe-associated molecular patterns (PAMPs/MAMPs) derived from microorganisms to initiate a series of basal immune responses called PAMP/MAMP-triggered immunity (PTI) (Boller and Felix, 2009; Boller and He, 2009; Cook et al., 2015). The PTI signaling events always involve a rapid $\mathrm{Ca}^{2+}$ influx, reactive oxygen species (ROS) production, activation of MAP ki-

$\dagger$ Contributed equally to this work

*Corresponding author (email: dplu@ @jziam.ac.cn) nase (MAPK) cascades, up-regulation of defense-related genes, callose deposition to strengthen the plant cell wall, and stomatal closure to prevent the entry of microbes (Boller and Felix, 2009; Boller and He, 2009; Cook et al., 2015).

Receptor-like kinases (RLKs) play important roles in plant innate immunity signaling (Wu and Zhou, 2013). The Arabidopsis genome encodes approximately 610 RLK/ Pelle/IRAK proteins, which also include receptor-like cytoplasmic kinases (RLCKs) (Shiu and Bleecker, 2001). RLKs contain a cytoplasmic kinase domain, a transmembrane domain, and an extracellular domain. In contrast, RLCKs lack 
the extracellular domain. As one of the best studied PRRs, FLS2 is an RLK with an extracellular leucine-rich repeat (LRR) domain (Gomez-Gomez and Boller, 2000). Through its LRR domain, FLS2 is able to perceive its corresponding PAMP, flg22, a conserved 22-amino-acid peptide from bacterial flagellin (Chinchilla et al., 2006). Together with another LRR-RLK, BAK1, FLS2 and flg22 form a PRR complex to initiate immunity signaling (Sun et al., 2013).

Recently, it has been reported that an RLCK, BIK1, plays a pivotal role in stomatal immunity signaling (Kadota et al., 2014; Li et al., 2014). BIK1 is a component of the FLS2 immune receptor complex (Lu et al., 2010; Zhang et al., 2010). It directly phosphorylates the NADPH oxidase RbohD at specific sites, resulting into ROS generation, thus causing the two guard cells of the stomata to actively reduce the opening aperture to prevent the entry of microbes (Kadota et al., 2014; Li et al., 2014). In addition to FLS2, BAK1 and BIK1, there are other RLKs found to be involved in PTI signaling. For examples, both EFR (the PRR of the elongation factor Tu) and CERK1 (the receptor of chitin) are RLKs (Miya et al., 2007; Zipfel et al., 2006). Similarly to BAK1, another LRR-RLK, suppressor of BIR1-1 (SOBIR1), has also been shown to be essential for the functioning of certain immune receptor complexes, but SOBIR1 prefers to work together with LRR-RLPs (receptor-like proteins) rather than RLKs (Liebrand et al., 2014). In addition, another plasma membrane localized RLCK, BSK1, physically associates with FLS2 and serves as a positive regulator of PTI signaling (Shi et al., 2013).

Most plant gram-negative bacterial pathogens can secrete effector proteins into host cells, where they target key components of the immune system to repress host defense (Feng and Zhou, 2012). As RLKs play important roles in immunity signaling, they serve as targets for a wide array of effectors. For example, a Pseudomonas syringae effector AvrPtoB, functions as an E3 ubiquitin ligase in plant cells and can target both FLS2 and CERK1 for degradation (Gohre et al., 2008; Gimenez-Ibanez et al., 2009). In addition, FLS2 is also targeted by AvrPto (Xiang et al., 2008). BIK1 serves as a target for AvrAC, an effector from Xanthomonas campestris pathovar campestris (Xcc) (Feng et al., 2012).

Xanthomonas spp. also possess a great number of T3Es, including the well-known TAL (transcription activationlike) effectors. TALs can activate the transcription of targeted plant genes by directly and specifically binding to their promoters thus promoting pathogenesis (Boch and Bonas, 2010). In addition to TAL effectors, Xanthomonas spp. also encode Xanthomonas outer proteins (Xops), which are non-TAL effector proteins. The functions of some Xops with known motifs from $X$. campestris pv. vesicatoria $(X c v)$, the causal agent of bacterial spot of tomato, have been characterized. For example, XopN interacts with tomato atypical receptor-like kinase1 (TARK1) to suppress immunity (Kim et al., 2009). XopD directly targets and de- sumoylates the tomato ethylene responsive transcription factor SIERF4 to repress ethylene-induced gene transcription that is required for plant immunity against $X c v$ (Kim et al., 2013). XopJ interacts with the plant proteasomal subunit RPT6 to inhibit host proteasome activity (Üstün et al., 2013).

Xanthomonas oryzae pv. oryzae (Xoo) is the causal agent of rice leaf blight disease. The Xoo strain MAFF311018 encodes 16 non-TAL effector proteins, while Philippine strain PXO99A encodes 18 (Furutani et al., 2009; Song and Yang, 2010). So far, the precise mechanisms of most non-TAL effectors remain largely unknown. Recently, a T3E XopR from the strain MAFF311018 was found to repress PTI responses in Arabidopsis, like inhibiting early defense gene expression and suppressing callose deposition (Akimoto-Tomiyama et al., 2012). This also suggested that Arabidopsis could be used as a host plant to study the roles of Xoo effectors. However, the precise target of XopR in plant cells and its mechanism of action are still unclear. The XopR genes in Xoo strains MAFF311018, KACC10331, and PXO99A are highly conserved, and they exhibit more than $99 \%$ homology to each other (Akimoto-Tomiyama et al., 2012). In our work, we studied XopR from the strain PXO99A and found that XopR targets a number of RLCKs including BIK1 and suppresses flg22-induced stomatal closure.

\section{RESULTS}

\section{XopR interacts with Arabidopsis BIK1}

Plant immune-related RLKs are targeted by a great number of effectors delivered from invading pathogens. Therefore, we hypothesized that there might be Xoo effectors that could also target plant immune-related RLKs. As most immune-related plant RLKs are mainly localized to the plasma membrane in plant cells, the potential RLK-targeting effectors from Xoo should also be localized to the plasma membrane. For this reason, we first analyzed the subcellular localization of Xoo effectors. Хoo effector genes fused with GFP tags were transiently expressed in protoplasts isolated from Arabidopsis leaf mesophyll cells and then subjected to confocal imaging. We found that most effectors analyzed, including XopL, XopV, XopC and XopW, were localized to the cytoplasm (Figure 1, Figure S1 in Supporting Information). Only XopR was localized to the plasma membrane. This is consistent with the finding reported by Akimoto-Tomiyama et al., who found that XopR from the strain MAFF311018 was also localized to the plasma membrane when it was transiently expressed in tobacco (Akimoto-Tomiyama et al., 2012). Moreover, as Xoo is the causal agent of rice leaf blight disease, we also analyzed the subcellular localization of XopR in rice protoplasts. Just as in Arabidopsis, XopR was also localized to the plasma membrane in rice (Figure 1B), further suggesting that XopR may 


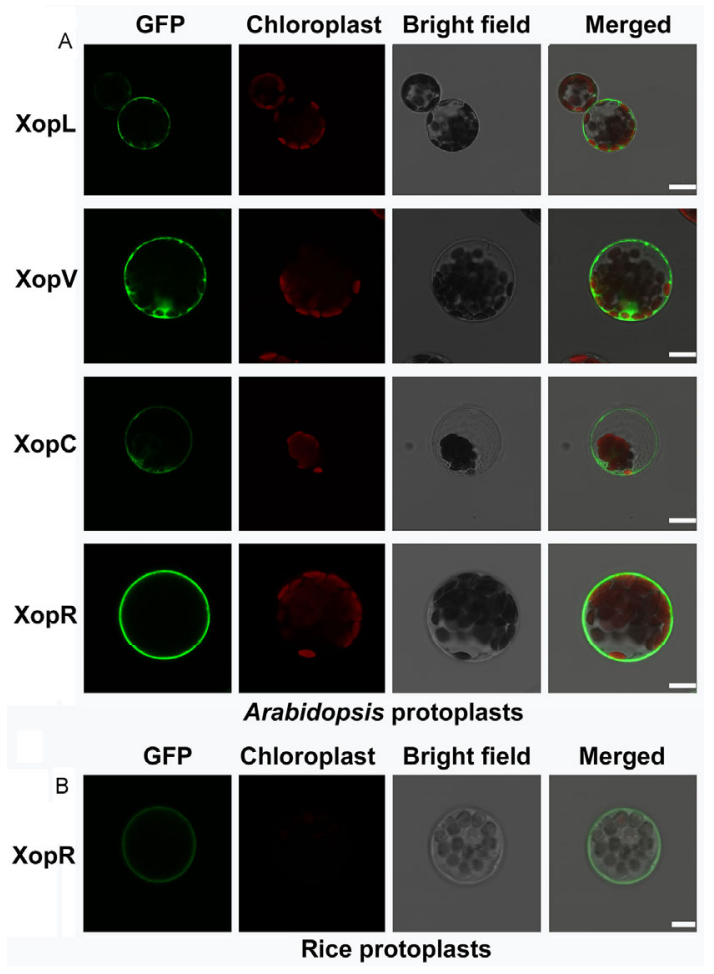

Figure 1 Subcellular localization of Xoo effectors in Arabidopsis and rice protoplasts. A, XopR localizes to the plasma membrane in Arabidopsis protoplasts. Хoo effector genes were expressed in Arabidopsis protoplasts then subjected to laser confocal imaging. XopL, XopV, and XopC localize primarily to the cytoplasm in Arabidopsis protoplasts, while XopR localizes specifically to the plasma membrane. Scale Bars=7.5 $\mu \mathrm{m}$. B, XopR also localizes to the plasma membrane in rice protoplasts. Chloroplasts were visualized using the autofluorescence of chlorophyll. Scale Bars $=5 \mu \mathrm{m}$.

function similarly in rice as in Arabidopsis.

To test whether PXO99A XopR can associate with any immune-related RLKs, we performed a co-immunoprecipitation (co-IP) analysis between XopR and the following three well-studied RLKs (including an RLCK): FLS2, BAK1 and BIK1. The results showed that XopR associated with BIK1, but not BAK1 or FLS2 (Figure 2A and B). To confirm these findings, we also performed a bimolecular fluorescence complementation assay (BiFC) in Arabidopsis protoplasts, where RLKs were tagged with the carboxylterminal half of YFP (yellow fluorescence protein; BIK1cYFP, BAK1-cYFP) and XopR was tagged with the amino-terminal half of YFP (XopR-nYFP). Consistently, the results showed that BIK1 associated with XopR at the plasma membrane in plant cells (Figure 2C). Moreover, recombinant His-FLAG-XopR could be pulled down by BIK1 protein fused to glutathione S-transferase (GST) in an in vitro GST pull-down assay (Figure 2D), further suggesting that XopR directly interacts with BIK1.

To test whether XopR can interact with RLCKs other than BIK1, we performed co-IP assays with XopR and PBL1, PBL2, PBL20, and PBS1 (members of the RLCK VII subfamily), BSK1 (a member of the RLCK subfamily
XII), and CDG1 (a subfamily VIIc member). The result showed that XopR strongly associated with PBL20 and CDG1, weakly associated with PBL2 and maybe PBL1 as well (Figure S2 in Supporting Information).

\section{BIK1 is not required for plasma-membrane localization of XopR}

BIK1 bears a myristoylation site, conferring its plasma membrane localization. Thus, when the second glycine $(\mathrm{G})$ is mutated to an alanine (A), BIK1 no longer localizes to the plasma membrane (Lu et al., 2010; Zhang et al., 2010). To test whether the plasma membrane localization of BIK1 is required for its association with XopR, we performed co-IP analysis between XopR and BIK1G2A, in which the second $\mathrm{G}$ was mutated to A. The results showed that XopR no longer associated with BIK1 when it lost its plasma membrane localization (Figure 3A), leading us to question whether the localization of XopR is dependent on BIK1. To test this, we expressed XopR-GFP in protoplasts isolated from the $B I K I$ gene null mutant plants. The results showed that XopR still localized to the plasma membrane in bikl, indicating that the localization of XopR is not dependent on BIK1 (Figure 3B).

To study the mechanism of XopR plasma membrane localization, we constructed a series of XopR truncations that were fused to GFP tags. Then, we transfected these XopR truncations into Arabidopsis protoplasts and observed their subcellular localization. The results showed that neither the $\mathrm{N}$-terminal fragment of XopR containing the first 49 amino acids, nor the $\mathrm{C}$-terminal fragment (from amino acid residues 142 to 437) localized to the plasma membrane. The XopR fragment containing amino acids 1-96 localized mainly to the plasma membrane, while the XopR fragment containing amino acids 1-144 localized completely to the plasma membrane, suggesting that the plasma membrane localization of XopR is dependent on its N-terminal 144 amino acids (Figure 3C).

\section{BIK1 phosphorylates XopR}

XopR is associated with BIK1, which is a kinase known to have strong autophosphorylation activity (Lu et al., 2010). Thus, we asked whether BIK1 could phosphorylate XopR. We performed an in vitro kinase assay using purified recombinant GST-BIK1 and GST-XopR proteins. The results, shown in Figure 4A, indicate that BIK1 has strong autophosphorylation activity, while the BIK1 kinase mutant $(\mathrm{BIK} 1 \mathrm{Km}), \mathrm{BIK} 1 \mathrm{~K} 105 \mathrm{E}$, which is deficient in ATP binding, has none. In the presence of ATP, BIK1 is able to phosphorylate XopR, as determined using an antiphospho-threonine antibody, indicating that at least certain threonine residues on XopR were phosphorylated by BIK1 (Figure 4A). This phosphorylation relies on BIK1 kinase activity because BIK1Km was not able to phosphorylate XopR (Figure 4 A).

Flg22 can induce BIK1 phosphorylation in protoplasts, which is exhibited by a mobility shift of BIK1 protein on 

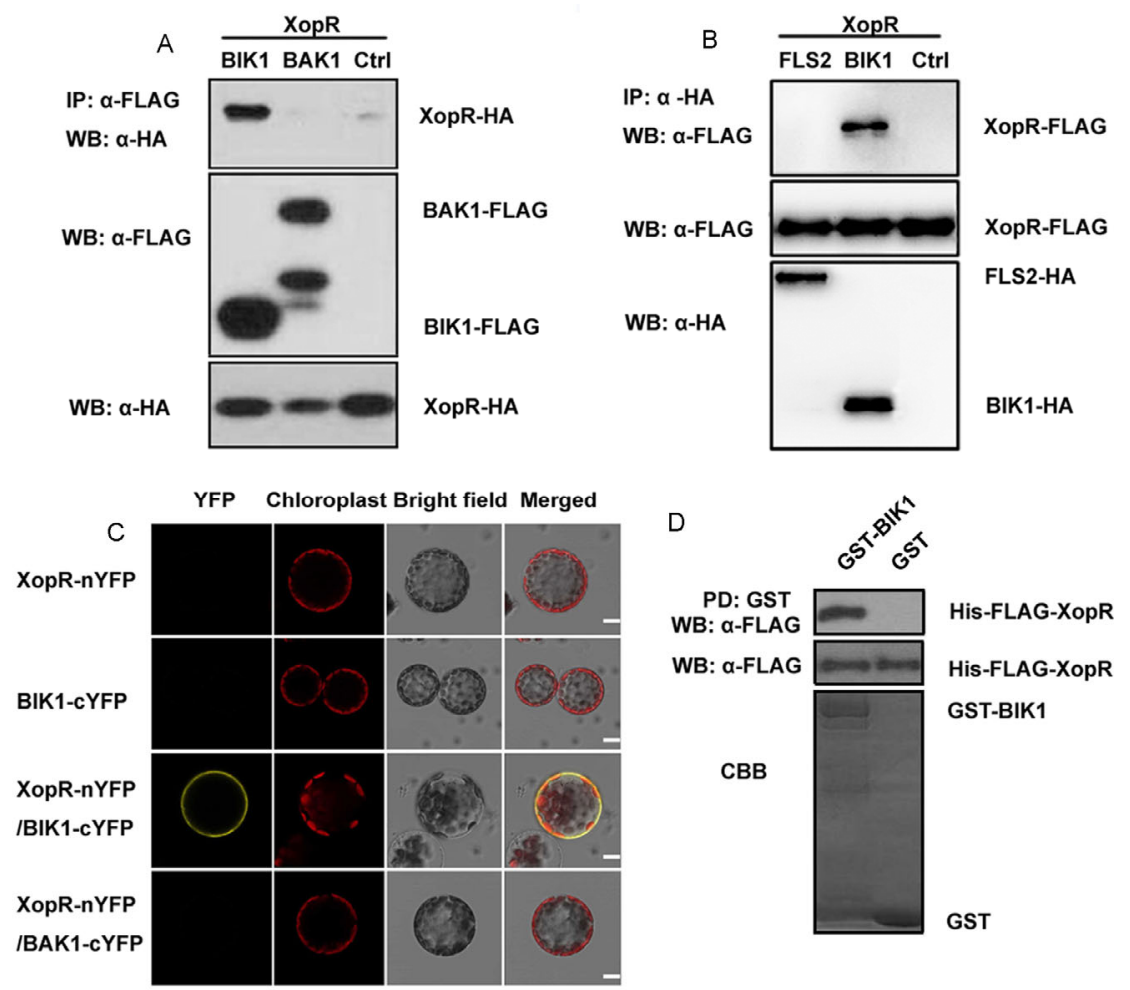

Figure 2 XopR interacts with BIK1. A, XopR associates with BIK1 but not BAK1 in Arabidopsis protoplasts. Receptor-like kinases (BIK1-FLAG, BAK1-FLAG) and XopR-HA were co-expressed in Arabidopsis protoplasts, and co-IP was performed using anti-FLAG antibodies. The associated proteins were analyzed by Western blot using an anti-HA antibody. B, XopR associates with BIK1 but not FLS2. Co-IP was carried out using protoplasts expressing RLKs-HA and XopR-Flag. C, The BiFC assays for XopR-BIK1 or XopR-BAK1 interaction in Arabidopsis protoplasts. The indicated BiFC constructs were transfected into protoplasts, and the fluorescence was visualized under a confocal microscope. nYFP, the amino-terminal half of yellow fluorescence protein (YFP), cYFP, the carboxyl-terminal half of YFP. Scale Bar=7.5 $\mu \mathrm{m}$. D, XopR interacts with BIK1 in vitro. The recombinant His-FLAG-XopR and GST-BIK1 were purified from Escherichia coli and used for a GST pull-down assay. The pulled down proteins were detected using an anti-FLAG antibody. $\mathrm{CBB}$, Coomassie brilliant blue staining.

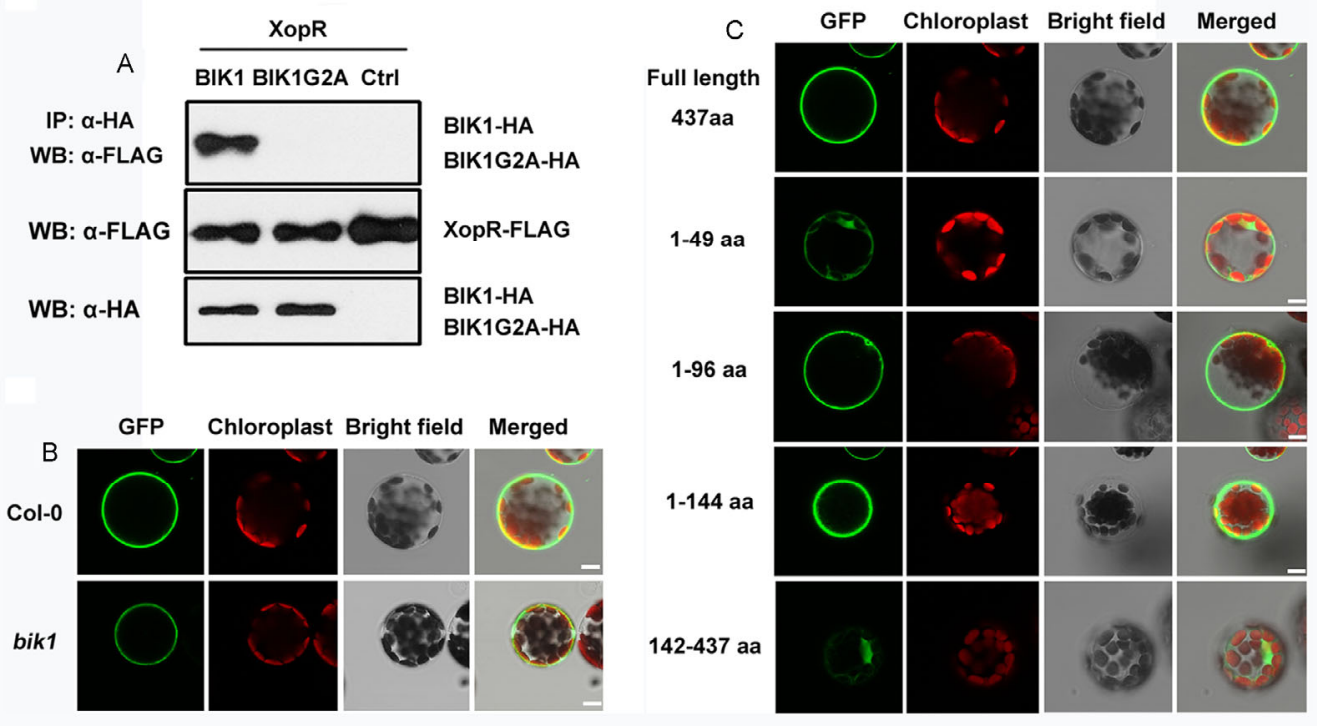

Figure 3 The plasma-membrane localization of XopR is independent of BIK1. A, XopR is not associated with BIK1G2A in Arabidopsis protoplasts. Protoplasts were co-transfected with the indicated constructs. BIK1-HA or BIK1G2A-HA proteins were immunoprecipitated using an anti-HA antibody, and the associated proteins were analyzed by Western blot using an anti-FLAG antibody. B, XopR-GFP localizes to the plasma membrane in bik1 protoplasts. C, The plasma-membrane localization of XopR is dependent on its N-terminal 144 amino acids. The different XopR truncations (indicated) were transfected into protoplasts, and the fluorescence was visualized under a confocal microscope. Chloroplasts were visualized using the autofluorescence of chlorophyll. Scale bar $=7.5 \mu \mathrm{m}$. 


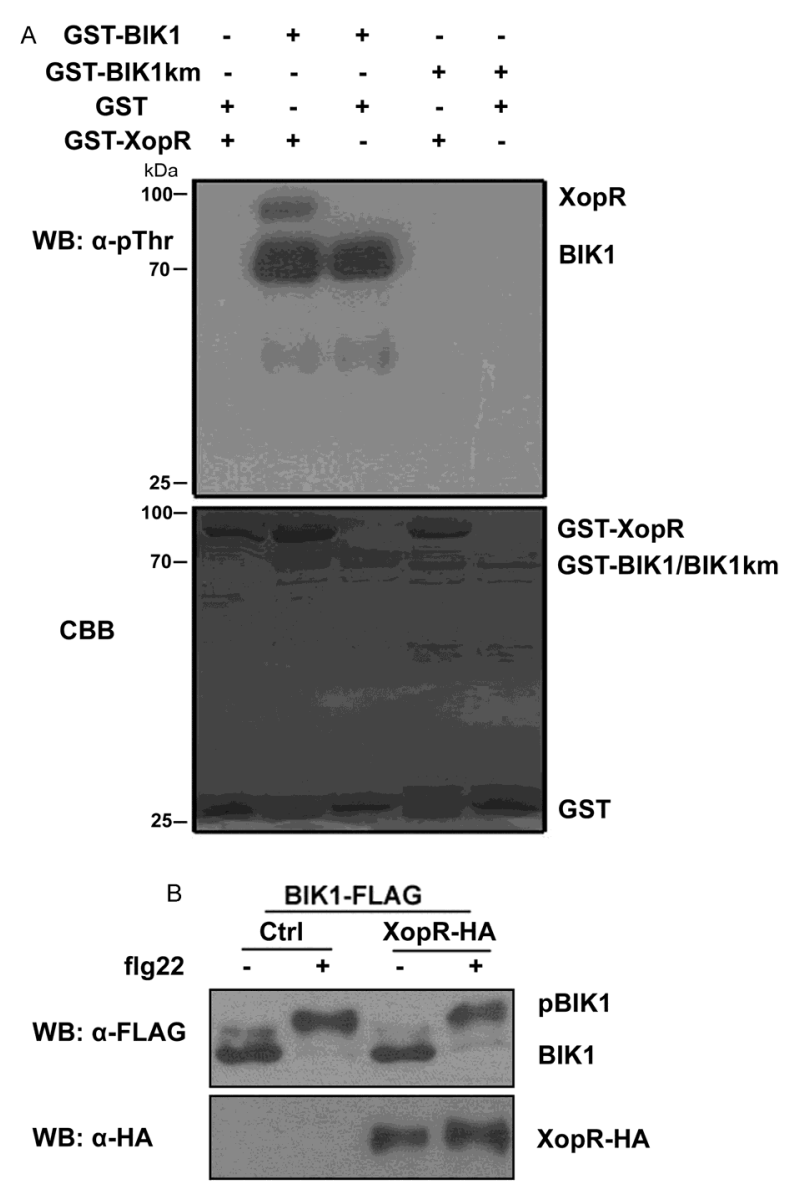

Figure 4 BIK1 phosphorylates XopR in vitro. A, XopR is phosphorylated by BIK1. Purified recombinant GST-XopR proteins were incubated with GST-BIK1 or GST-BIK1Km in the presence of ATP, and the phosphorylation was detected by Western blot using an anti-phospho-Thr (pThr) antibody. CBB, Coomassie brilliant blue staining. B, XopR does not inhibit flg22-induced BIK1 phosphorylation. Protoplasts were co-transfected with BIK1-FLAG and XopR-HA and used for Western blot analysis. Before harvesting, the protoplasts were treated with $1 \mu \mathrm{mol} \mathrm{L}{ }^{-1}$ flg 22 for $10 \mathrm{~min}$.

SDS-PAGE (Lu et al., 2010; Zhang et al., 2010). It has been reported that an Xcc effector, AvrAC, can target BIK1 and inhibit BIK1 kinase activity. Additionally, flg22-induced BIK1 phosphorylation is blocked by AvrAC in protoplasts (Feng et al., 2012). Thus, if an effector inhibits BIK1 kinase activity, it might also suppress the flg22-induced BIK1 phosphorylation. To test the effect of XopR on BIK1 phosphorylation, we co-expressed BIK1 and XopR in protoplasts. The result shows that although XopR associates with BIK1, it dose not affect flg22-induced BIK1 phosphorylation (Figure 4B).

BIK1 phosphorylates the NADPH oxidase RbohD to regulate ROS generation (Kadota et al., 2014; Li et al., 2014). To investigate whether XopR can interfere with the phosphorylation of RbohD by BIK1, we performed an in vitro kinase assay followed by an acrylamide-pendant Phos-tag ${ }^{\text {TM }}$ SDS-PAGE analysis. Consistently, BIK1 phosphorylated the N-terminal fragment of RbohD, His-FLAG-
RbohDNT, as demonstrated by the slowly migrating forms of RbohDNT on Phos-tag ${ }^{\mathrm{TM}}$ SDS-PAGE (Figure S3 in Supporting Information) (Li et al., 2014). When XopR was added to the reaction, it seemed that the phosphorylation of RbohDNT by BIK1 was decreased, but only to a very slight extent (Figure S3 in Supporting Information).

\section{XopR suppresses PAMP-induced stomatal closure}

BIK1 is involved in PAMP-triggered stomatal closure (Kadota et al., 2014; Li et al., 2014), and XopR targets BIK1. Therefore, we asked whether XopR could interfere with PAMP-triggered stomatal closure. Because XopR functions similarly in rice and in Arabidopsis (Akimoto-Tomiyama et al., 2012), we generated two independent transgenic lines (3-9 and 6-1) expressing full-length XopR in Arabidopsis ecotype Col-0. XopR was fused to a C-terminal hemagglutinin (HA) tag and was under the control of the XVEinducible promoter. XVE-induced XopR expression was detected in transgenic lines by Western blotting with an anti-HA antibody (Figure 5B). Then, we performed a stomatal aperture assay and found that the expression of XopR suppressed flg22-triggered stomatal closure (Figure 5A). In addition, we also tested the effect of XopR on other PTI signaling events, such as MAP kinase (MAPK) activation and ROS production. As shown in Figure 5C, XopR expression did not affect flg22-induced MAPK6/3/4 phosphorylation. In fact, even in the bikl mutant, flg22-induced MAPK activation was not compromised (Zhang and Zhou, 2010). As for ROS production, flg22-induced $\mathrm{H}_{2} \mathrm{O}_{2}$ production in bikl plants was significantly compromised, as reported by $\mathrm{Li}$ et al. (Figure $4 \mathrm{~S}$ in Supporting Information) (Li et al., 2014). However, flg22-induced $\mathrm{H}_{2} \mathrm{O}_{2}$ production in the two XopR transgenic lines was only slightly compromised and was overall very close to that in wild-type plants (Figure $4 \mathrm{~S}$ in Supporting Information).

\section{DISCUSSION}

XopR from the Xoo strain MAFF311018 has been shown to suppress PAMP-triggered immune responses in Arabidopsis (Akimoto-Tomiyama et al., 2012); however, the target of XopR was still unknown. Here, we determined that XopR from the strain PXO99A associates with BIK1 and other RLCKs in plant cells (Figure 2, Figure S2 in Supporting Information). BIK1 is a component of the FLS2 immune receptor complex (Lu et al., 2010; Zhang et al., 2010) and directly phosphorylates the NADPH oxidase RbohD at specific sites, thus to control ROS generation and stomatal immunity (Kadota et al., 2014; Li et al., 2014). In transgenic Arabidopsis expressing XopR, we found that XopR suppresses flg22-induced stomatal closure (Figure 5A). When an effector binds to a plant immunity signaling component, it can affect some aspects of the target. In this work we show that although XopR interacts with BIK1, it does not affect flg22-induced BIK1 phosphorylation (Figure 4B). In 

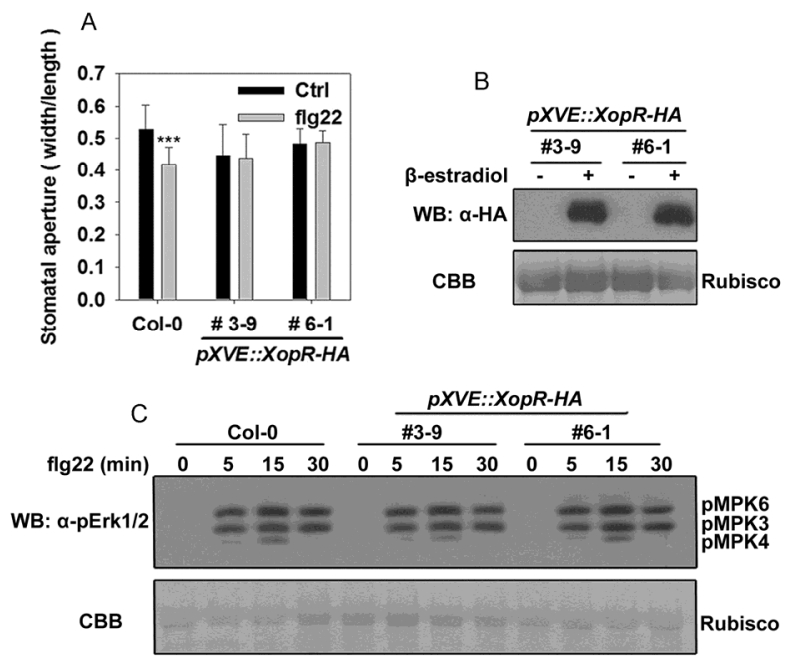

Figure 5 Suppression of PAMP-triggered stomatal closure by XopR. A, XopR expression suppresses flg22-induced stomatal closure. Both Arabidopsis Col-0 and two XopR transgenic lines (\#3-9 and \#6-1) were pretreated with $\beta$-estradiol or a mock solution, and then used to measure flg22-induced stomatal closure. The experiments were repeated three times. Error bars indicate $\pm \mathrm{SE}$ based on $n \geqslant 30$ stomata from at least three independent plants. Statistical significance compared to controls was determined using a Student's $t$-test: $* * *, P<0.001$. B, Confirmation of XopR-HA expression in the two transgenic lines. Plants were treated with $4 \mu \mathrm{mol} \mathrm{L}{ }^{-1} \beta$-estradiol or a mock solution for $12 \mathrm{~h}$ and then were used in Western blot using an anti-HA antibody. C, Flg22-induced MAPK activation in wild-type and two XopR transgenic lines. The plants were treated with $100 \mathrm{nmol} \mathrm{L}^{-1}$ flg22 for indicated time. The phosphorylated MAPKs were detected using an anti-Erk1/2 antibody. Coomassie Blue stained Rusbisco proteins were used as a loading control.

addition, XopR may repress the phosphorylation of RbohD by BIK1, but only slightly (Figure S3 in Supporting Information). Moreover, flg22-induced $\mathrm{H}_{2} \mathrm{O}_{2}$ production in the XopR transgenic lines was only marginally compromised relative to that in wild-type plants (Figure S4 in Supporting Information). Taken together, our results suggest that XopR likely only slightly, or does not, affect the ability of BIK1 to phosphorylate RhobD and the BIK1-mediated ROS production. This seems counterintuitive, as XopR obviously suppresses flg22-induced stomatal closure (Figure 5A). In fact, it has been proposed that BIK1 may regulate additional signaling components downstream of ROS production during stomatal immunity (Arnaud and Hwang, 2015; Li et al., 2014), because $\mathrm{H}_{2} \mathrm{O}_{2}$ only partially induces stomatal closure in bikl and bikl pbll plants (Li et al., 2014). Thus, it is likely that XopR might inhibit a novel and as-yet unidentified role that BIK1 plays downstream of ROS production. In addition, our results show that XopR associates with various RLCKs other than BIK1 (Figure S2 in Supporting Information), suggesting that other components targeted by XopR may also be involved in PAMP-triggered stomatal closure.

We also show that BIK1 phosphorylates XopR (Figure 4A). The phosphorylation of XopR by BIK1 in vitro also confirms their direct interaction. Indeed, effector phosphor- ylation also occurs in other pathogen-host interactions. It has been reported that AvrPto is phosphorylated when expressed in plant leaves. The substitution of phosphorylation sites in AvrPto significantly decreased the ability of AvrPto to enhance disease symptoms in susceptible tomato leaves (Anderson et al., 2006). Similarly, AvrPtoB is also phosphorylated in plants, and this phosphorylation is required for virulence (Xiao et al., 2007). Thus, it is believed that AvrPto and AvrPtoB have evolved to mimic the substrates of certain highly conserved plant kinases to enhance virulence activity (Anderson et al., 2006; Xiao et al., 2007). In our work, we found that XopR could be phosphorylated by BIK1 in vitro, but further studies are needed to identify the sites on XopR that are phosphorylated by BIK1 and to elucidate the role of this phosphorylation.

When delivered to or expressed in plant cells, a large number of bacterial effectors localize to the plasma membrane through their N-myristoylation motifs, including Pst effectors AvrB, AvrRpm1, AvrPphB, AvrPto, and HopF2 (Göhre et al., 2008; Nimchuk et al., 2000; RobertSeilaniantz et al., 2006; Shan et al., 2000; Xiang et al., 2008). XopR also localizes to the plasma membrane in plant cells. It was recently suggested that the coiled-coil (CC) domains of MAFF 311018 XopR between amino acid residues 271 and 300 might contribute to its plasma membrane localization (Akimoto-Tomiyama et al., 2012). However, in our work, we found that the first 144 amino acids of XopR were sufficient to target it to the plasma membrane (Figure $3 \mathrm{C})$. Therefore, it is possible that there are certain unidentified motifs in this region that enable the plasma-membrane localization of XopR. Further work will be needed to identify such motifs and to investigate their mechanisms of action.

\section{MATERIALS AND METHODS}

\section{Plant materials and growth conditions}

Wild-type (Col-0), bikl mutant and XopR transgenic Arabidopsis plants were grown in a growth chamber at $22^{\circ} \mathrm{C}$ with $60 \%$ relative humidity, $70 \mu \mathrm{E} \mathrm{m}^{-2} \mathrm{~s}^{-1}$ light, and a $12-\mathrm{h}$ photoperiod for 4 weeks before protoplast isolation, stomatal assays, and other studies.

\section{Plasmid construction and generation of transgenic plants}

Arabidopsis BAK1, BIK1, BIK1G2A, BIK1Km (BIK1K105E) and FLS2 constructs were reported previously by Lu et al. (Lu et al., 2010). Effector genes were amplified by PCR from PXO99A genomic DNA and cloned into plant expression vectors. $X o p R, B I K 1$, and $B A K 1$ were sub-cloned into the modified BiFC vectors. Full-length $X o p R$ was subcloned into a GST fusion protein expression vector pGEX4T-1, or a His-tag fusion protein expression 
Table 1 Oligo primers used in this study

\begin{tabular}{ll}
\hline Name & Sequence $\left(5^{\prime} \rightarrow 3^{\prime}\right)$ \\
\hline XopR-BamHI-F & CGCGGATCCATGCGCACGAATTTTCT \\
XopR-StuI-R & GAAGGCCTTCGGTAACCGTTCTCCAT \\
XopL-BamHI-F & CGGGATCCATGAGGAAGGCGATG \\
XopL-StuI-R & AAAAGGCCTGCGCGAAGGTTCCGA \\
XopV-BamHI-F & CGGGATCCATGCGCCGAAGCGCATCA \\
XopV-StuI-R & GAAGGCCTTTCACCGTTAGGGTCAGAATGTG \\
XopC-BamHI-F & CGGGATCCATGGTCGGGATGCTCGAT \\
XopC-StuI-R & TCCCCCGGGTTTCCGGCTTTCTCAA \\
XopR-XhoI-F & CCGCTCGAGATGCGCACGAATTTTCT \\
XopR-SpeI-R & GGACTAGTTCAAGCGTAGTCTGGAACGTCGTATGGGTAACCAGCGTAGTCTGGAACGTCGTATGGGTATCGGTAACCGT \\
XopR-StuI-R1 & GATCC \\
XopR-StuI-R2 & GAAGGCCTGGCGTGGGGTGTGGAT \\
XopR-StuI-R3 & GAAGGCCTTCTAGTACGTGCAGGCG \\
XopR-BamHI-F1 & CGCGGATCCATGCGTACTAGAGAGCCC \\
\hline
\end{tabular}

vector pET28a. The XopR transgenic plants were generated by Agrobacterium tumefaciens-mediated transformation in Col-0 using the XVE vector harboring the XopR gene fused to a C-terminal HA tag under the control of the XVE-inducible promoter (Zuo et al., 2000). The transgenic plants were confirmed by Western blot with an anti-HA antibody (Sigma, USA). The primer sequences for all experiments are listed in Table 1.

\section{Transient gene expression and BiFC assays in Arabidop- sis or rice protoplasts}

Protoplast transfections were carried out as described (He et al., 2006). Rice protoplasts were isolated as described (Zhang et al., 2011). Protoplasts were transfected with transient gene expression vectors or BiFC vectors harboring effector genes or various RLK genes, and the fluorescence was detected using a Leica confocal laser scanning microscope.

\section{Co-immunoprecipitation (co-IP) assays}

Co-IP assays were performed as previously described (Lu et al., 2010).

\section{Recombinant protein expression and GST pull-down assay}

The recombinant proteins were purified from Escherichia coli according to the manufacturer's instructions. The GST-pull down assay was carried out as described (Feng et al., 2012). The bound His-FLAG-XopR proteins were detected by Western blot using an anti-FLAG antibody (Sigma).

\section{In vitro phosphorylation assays}

Kinase reactions were performed as described (Lin et al., 2014). Protein phosphorylation was detected by an antiphospho-Threonine antibody (Cell Signaling Technology, USA).

\section{Stomatal aperture measurements}

The method used for the stomatal aperture assay was modified from $\mathrm{Li}$ et al. ( $\mathrm{Li}$ et al., 2014). Both Arabidopsis Col-0 and XopR transgenic plants were pretreated with $4 \mu \mathrm{mol} \mathrm{L}{ }^{-1}$ $\beta$-estradiol or a mock solution for $12 \mathrm{~h}$. Then, plants were kept under light for $2 \mathrm{~h}$ to ensure that most of the stomata were opened before flg22 (EZBiolab, USA) treatment. Leaves of 4-week-old plants were collected and floated in buffer (10 mmol L $\mathrm{m}^{-1} \mathrm{MES}$ ( $\mathrm{pH} 6.15$ ), $10 \mathrm{mmol} \mathrm{L}^{-1} \mathrm{KCl}$, $10 \mathrm{mmol} \mathrm{L}^{-1} \mathrm{CaCl}_{2}$ ). The stomata on the leaf's abaxial epidermis were observed using a microscope (Leica, Germany) after the leaves were treated with $10 \mu \mathrm{mol} \mathrm{L}^{-1}$ flg 22 for $1 \mathrm{~h}$. The stomatal aperture was measured using the Image $\mathrm{J}$ software (NIH, USA).

\section{MAPK activity assays}

MAPK activity assays of wild-type and XopR transgenic plants were performed as previously described (Feng et al., 2012).

Compliance and ethics The author(s) declare that they have no conflict of interest.

Acknowledgements We thank Drs. Libo Shan and Ping He from Texas A\&M University for kindly providing us with constructs including BIK1, BIK1G2A, BIK1Km, FLS2, BAK1 in various vectors and bik1 mutant seeds. This work was supported by the National Natural Science Foundation of China (31322009), the National Basic Research Program of China (2015CB910200), and the State Key Laboratory of Plant Genomics of China (SKLPG2011B0301, SKLPG2011A0301).

Akimoto-Tomiyama, C., Furutani, A., Tsuge, S., Washington, E.J., Nishizawa, Y., Minami, E., and Ochiai, H. (2012). XopR, a type III effector secreted by Xanthomonas oryzae pv. oryzae, suppresses microbe-associated molecular pattern-triggered immunity in Arabidopsis thaliana. Mol Plant Microbe Interact 25, 505-514.

Anderson, J.C., Pascuzzi, P.E., Xiao, F., Sessa, G., and Martin, G.B. (2006). Host-mediated phosphorylation of type III effector AvrPto promotes Pseudomonas virulence and avirulence in tomato. Plant Cell 
$18,502-514$.

Arnaud, D., and Hwang, I. (2015). A sophisticated network of signaling pathways regulates stomatal defenses to bacterial pathogens. Mol Plant $8,566-581$.

Boch, J., and Bonas, U. (2010). Xanthomonas AvrBs3 family-type III effectors: discovery and function. Annu Rev Phytopathol 48, 419-436.

Boller, T., and Felix, G. (2009). A renaissance of elicitors: perception of microbe-associated molecular patterns and danger signals by pattern-recognition receptors. Annu Rev Plant Biol 60, 379-406.

Boller, T., and He, S.Y. (2009). Innate immunity in plants: an arms race between pattern recognition receptors in plants and effectors in microbial pathogens. Science 324, 742-744.

Chinchilla, D., Bauer, Z., Regenass, M., Boller, T., and Felix, G. (2006). The Arabidopsis receptor kinase FLS2 binds flg22 and determines the specificity of flagellin perception. Plant Cell 18, 465-476.

Cook, D.E., Mesarich, C.H., and Thomma, B.P. (2015). Understanding plant immunity as a surveillance system to detect invasion. Annu Rev Phytopathol 53, 541-563.

Feng, F., Yang, F., Rong, W., Wu, X., Zhang, J., Chen, S., He, C., and Zhou, J.M. (2012). A Xanthomonas uridine 5'-monophosphate transferase inhibits plant immune kinases. Nature 485, 114-118.

Feng, F., and Zhou, J.M. (2012). Plant-bacterial pathogen interactions mediated by type III effectors. Curr Opin Plant Biol 15, 469-476.

Furutani, A., Takaoka, M., Sanada, H., Noguchi, Y., Oku, T., Tsuno, K., Ochiai, H., and Tsuge, S. (2009). Identification of novel type III secretion effectors in Xanthomonas oryzae pv. oryzae. Mol Plant Microbe Interact 22, 96-106.

Gimenez-Ibanez, S., Hann, D.R., Ntoukakis, V., Petutschnig, E., Lipka, V., and Rathjen, J.P. (2009). AvrPtoB targets the LysM receptor kinase CERK1 to promote bacterial virulence on plants. Curr Biol 19, 423-429.

Göhre, V., Spallek, T., Häweker, H., Mersmann, S., Mentzel, T., Boller, T., de Torres, M., Mansfield, J.W., and Robatzek, S. (2008). Plant pattern-recognition receptor FLS2 is directed for degradation by the bacterial ubiquitin ligase AvrPtoB. Curr Biol 18, 1824-1832.

Gomez-Gomez, L., and Boller, T. (2000). FLS2: an LRR receptor-like kinase involved in the perception of the bacterial elicitor flagellin in Arabidopsis. Mol Cell 5, 1003-1011.

He, P., Shan L., Lin, N.C., Martin, G.B., Kemmerling, B., Nürnberger, T., and Sheen, J. (2006). Specific bacterial suppressors of MAMP signaling upstream of MAPKKK in Arabidopsis innate immunity. Cell 125, 563-575.

Kadota, Y., Sklenar, J., Derbyshire, P., Stransfeld, L., Asai, S., Ntoukakis, V., Jones, J.D., Shirasu, K., Menke, F., Jones, A., and Zipfel, C. (2014). Direct regulation of the NADPH oxidase RBOHD by the PRR-associated kinase BIK1 during plant immunity. Mol Cell 54, 43-55.

Kim, J.G., Li, X., Roden, J.A., Taylor, K.W., Aakre, C.D., Su, B., Lalonde, S., Kirik, A., Chen, Y., Baranage, G., McLane, H., Martin, G.B., and Mudgett, M.B. (2009). Xanthomonas T3S effector XopN suppresses PAMP-triggered immunity and interacts with a tomato atypical receptor-like kinase and TFT1. Plant Cell 21, 1305-1323.

Kim, J.G., Stork, W., and Mudgett, M.B. (2013). Xanthomonas type III effector XopD desumoylates tomato transcription factor SIERF4 to suppress ethylene responses and promote pathogen growth. Cell Host Microbe 13, 143-154.

Li, L., Li, M., Yu, L., Zhou, Z., Liang, X., Liu, Z., Cai, G., Gao, L., Zhang, X., Wang, Y., Chen, S., and Zhou, J.M. (2014). The FLS2-associated kinase BIK1 directly phosphorylates the NADPH oxidase RbohD to control plant immunity. Cell Host Microbe 15, 329-338.

Liebrand, T.W., van den Burg, H.A., and Joosten, M.H. (2014). Two for all: receptor-associated kinases SOBIR1 and BAK1. Trends Plant Sci 19, 123-132.

Lin, W., Li, B., Lu, D., Chen, S., Zhu, N., He, P., and Shan, L. (2014). Tyrosine phosphorylation of protein kinase complex BAK1/BIK1 me- diates Arabidopsis innate immunity. Proc Natl Acad Sci USA 111, 3632-3637.

Lu, D., Wu, S., Gao, X., Zhang, Y., Shan, L., and He, P. (2010). A receptor-like cytoplasmic kinase, BIK1, associates with a flagellin receptor complex to initiate plant innate immunity. Proc Natl Acad Sci USA 107, 496-501.

Miya, A., Albert, P., Shinya, T., Desaki, Y., Ichimura, K., Shirasu, K., Narusaka, Y., Kawakami, N., Kaku, H., and Shibuya, N. (2007). CERK1, a LysM receptor kinase, is essential for chitin elicitor signaling in Arabidopsis. Proc Natl Acad Sci USA 104, 19613-19618.

Nimchuk, Z., Marois, E., Kiemtrup, S., Leister, R.T., Kataqiri, F., and Dangl, J.L. (2000). Eukaryotic fatty acylation drives plasma membrane targeting and enhances function of several type III effector proteins from Pseudomonas syringae. Cell 101, 353-363.

Robert-Seilaniantz, A., Shan, L., Zhou, J.M., and Tang,X.Y. (2006). The Pseudomonas syringae pv. tomato DC3000 type III effector HopF2 has a putative myristoylation site required for its avirulence and virulence functions. Mol Plant Microbe Interact 19, 130-138.

Shan, L., Thara, V.K., Martin, G.B., Zhou, J. M., and Tang, X.Y. (2000). The pseudomonas AvrPto protein is differentially recognized by tomato and tobacco and is localized to the plant plasma membrane. Plant Cell 12, 2323-2338.

Shi, H., Shen, Q., Qi, Y., Yan, H., Nie, H., Chen, Y., Zhao, T., Katagiri, F., and Tang, D. (2013). BR-SIGNALING KINASE1 physically associates with FLAGELLIN SENSING2 and regulates plant innate immunity in Arabidopsis. Plant Cell 25, 1143-1157.

Shiu, S.H., and Bleecker, A.B. (2001). Receptor-like kinases from Arabidopsis form a monophyletic gene family related to animal receptor kinases. Proc Natl Acad Sci USA 98, 10763-10768.

Song, C., and Yang, B. (2010). Mutagenesis of 18 type III effectors reveals virulence function of XopZ (PXO99) in Xanthomonas oryzae pv. oryzae. Mol Plant Microbe Interact 23, 893-902.

Sun, Y., Li, L., Macho, A.P., Han, Z., Hu, Z., Zipfel, C., Zhou, J.M., and Chai, J. (2013). Structural basis for flg22-induced activation of the Arabidopsis FLS2-BAK1 immune complex. Science 342, 624-628.

Üstün, S., Bartetzko, V., and Börnke, F. (2013). The Xanthomonas campestris type III effector XopJ targets the host cell proteasome to suppress salicylic-acid mediated plant defence. PLoS Pathog 9, e1003427.

Wu, Y., and Zhou, J.M. (2013). Receptor-like kinases in plant innate immunity. J Integr Plant Biol 55, 1271-1286.

Xiang, T., Zong, N., Zou, Y., Wu, Y., Zhang, J., Xing, W., Li, Y., Tang, X., Zhu, L., Chai, J., and Zhou, J.M. (2008). Pseudomonas syringae effector AvrPto blocks innate immunity by targeting receptor kinases. Curr Biol 18, 74-80.

Xiao, F., Giavalisco, P., and Martin, G.B. (2007). Pseudomonas syringae type III effector AvrPtoB is phosphorylated in plant cells on serine 258, promoting its virulence activity. J Biol Chem 282, 30737-30744.

Zhang, J., Li, W., Xiang, T., Liu, Z., Laluk, K., Ding, X., Zou, Y., Gao, M., Zhang, X., Chen, S., Mengiste, T., Zhang, Y., Zhou, J.M. (2010). Receptor-like cytoplasmic kinases integrate signaling from multiple plant immune receptors and are targeted by a Pseudomonas syringae effector. Cell Host Microbe 7, 290-301.

Zhang, J., and Zhou, J.M. (2010). Plant immunity triggered by microbial molecular signatures. Mol Plant 3, 783-793.

Zhang, Y., Su, J.B., Duan, S., Ao, Y., Dai, J.R., Liu, J., Wang, P., Li, Y.G., Liu, B., Feng, D.R., Wang, J.F., and Wang, H.B. (2011). A highly efficient rice green tissue protoplast system for transient gene expression and studying light/chloroplast-related processes. Plant Methods 7, 30.

Zipfel, C., Kunze, G., Chinchilla, D., Caniard, A., Jones, J.D.G., Boller, T., and Felix, G. (2006). Perception of the bacterial PAMP EF-Tu by the receptor EFR restricts Agrobacterium-mediated transformation. Cell $125,749-760$.

Zuo, J.R., Niu, Q.W., and Chua, N.H. (2000). An estrogen receptor-based transactivator XVE mediates highly inducible gene expression in transgenic plants. Plant J 24, 265-273.

Open Access This article is distributed under the terms of the Creative Commons Attribution License which permits any use, distribution, and reproduction in any medium, provided the original author(s) and source are credited. 


\section{SUPPORTING INFORMATION}

Figure S1 Subcellular localization of XopW in Arabidopsis protoplasts. Laser confocal images show the Xoo effector XopW is localized primarily to the cytoplasm in Arabidopsis protoplasts. Scale bar=7.5 $\mu \mathrm{m}$.

Figure S2 XopR associates with a number of RLCKs in Arabidopsis protoplasts. Various RLCKs (PBL1-FLAG, PBL2-FLAG, PBL20-FLAG, PBS1-FLAG, BSK1-FLAG, CDG1-FLAG, or a control vector) and XopR-HA were co-expressed in Arabidopsis protoplasts, and co-IP was performed using anti-FLAG antibodies. The associated proteins were analyzed by Western blot using an anti-HA antibody.

Figure S3 The effect of XopR on the phosphorylation of RbohD by BIK1. The purified recombinant protein His-FLAG-RbohDNT (the N-terminal fragment of RbohD) was incubated with GST-BIK1 in the presence of ATP. Then, gradually increasing amounts of XopR protein (from 0-25 $\mu \mathrm{g}$ ) was applied to individual reactions. The phosphorylated His-FLAG-RbohDNT proteins were separated from unphosphorylated ones on an acrylamide-pendant Phos-tag ${ }^{\mathrm{TM}}$ SDS-PAGE. The RbohDNT proteins were detected by Western blot using an anti-FLAG antibody.

Figure S4 The effect of XopR on flg22-induced ROS burst. Flg22-induced $\mathrm{H}_{2} \mathrm{O}_{2}$ production was measured in wild-type and XopR transgenic plants, and three biological replicates were performed. Data are represented as the mean \pm SE. RLU, relative luminescence units.

The supporting information is available online at life.scichina.com and link.springer.com. The supporting materials are published as submitted, without typesetting or editing. The responsibility for scientific accuracy and content remains entirely with the authors. 